\title{
Pharmacist Prescriptive Authority for Acne: An Evidence-Based Approach to Policy
}

Jennifer Athay Adams, PharmD, EdD ${ }^{1}$; Alex J. Adams, PharmD, MPH ${ }^{2}$; Michael E. Klepser, PharmD, FCCP

${ }^{1}$ Idaho State University, College of Pharmacy; ${ }^{2}$ Idaho State Board of Pharmacy; ${ }^{3}$ Ferris State University, College of Pharmacy

\begin{abstract}
The diagnosis of acne is typically straightforward and based on physical signs and symptoms. Some jurisdictions in Canada, the United Kingdom, and United States have enabled a pharmacist treatment model to diagnose and manage patients with mild acne using prescription medications. Studies have found the model to be safe and effective, while simultaneously increasing more timely access to care for patients which may reduce the potential adverse impacts of acne. Further, use of a standardized protocol may alleviate some of the concerns expressed over the model. This paper summarize answers to frequent questions to help policymakers consider the objective evidence for their jurisdiction.
\end{abstract}

\section{Pharmacist Management of Acne: An Evidence-Based Approach}

\section{Background}

Acne is a chronic inflammatory skin disease that affects up to 50 million Americans annually. ${ }^{1-2}$ While acne can occur at any age, it most commonly begins in puberty and affects an estimated $85 \%$ of people between the ages of 12 and $24 .^{2-3}$ Acne manifests as skin lesions on the face, neck, chest, upper arms and back, and as a result, can cause significant embarrassment, anxiety, depression, and low self-esteem in patients. ${ }^{3-5}$ Moreover, acne can lead to scarring. ${ }^{6-7}$ The diagnosis of acne is typically straightforward and based on physical signs and symptoms. ${ }^{8}$

In 2013, acne accounted for $\$ 1.2$ billion in medical costs, including $\$ 400$ million in lost productivity among patients and caregivers. ${ }^{9}$ Despite these costs, acne remains undertreated. Moreover, many adolescents delay seeking treatment, or seek medical assistance only with more severe acne. ${ }^{10-12}$ Access to care for acne is compounded by the nationwide shortage of physicians generally, and dermatologists specifically, and the uneven geographic distribution of dermatologists in the United States. ${ }^{13}$

As a result, some have called for new ways to reduce barriers to access for patients with acne. Some jurisdictions have enabled a pharmacist treatment model to manage patients with mild acne using prescription medications. This pharmacistled model has been explored in Canada14-16, the United Kingdom $^{17-20}$, and the United States ${ }^{21-23}$. This paper aims to summarize answers to the frequently asked questions we have received on this topic by using objective evidence from the literature.

Corresponding Author: Alex J. Adams, PharmD, MPH

Former Executive Director

Idaho State Board of Pharmacy

4537 N Molly Way, Meridian, ID 83646

Email: alexadamsrph@gmail.com

\section{Key Areas of Concern}

Pharmacists are not trained diagnosticians. How will they identify acne relative to other skin conditions?

Dermatologists have noted that acne is generally "easy to diagnose" though some work is necessary to classify its severity to determine an appropriate course of treatment. ${ }^{24-25}$ Acne classification schemes are not universally accepted, but the condition is commonly differentiated as mild, moderate, or severe, depending on the types of lesions and their frequency. ${ }^{1}$ Patients often successfully self-diagnose and manage mild to moderate acne. In a study of 18 females and three males, $100 \%$ accurately self-diagnosed their condition, with high concordance of lesion type and count with the investgators. ${ }^{25}$ Another study found self-diagnosis validity was moderate, with results improving as subjects' total amount of observable acne increased. ${ }^{26}$

Overall, with the reported accuracy of patient self-diagnosis, it is plausible that trained pharmacists can assist in diagnosing mild to moderate acne, while referring patients to other health professionals when appropriate. Further, given that patients routinely seek over-the-counter medications and self-treat acne more frequently than consulting a physician, a pharmacist management model may increase access to a broader array of medications that may be more appropriate for specific patients. Current accreditation standards for U.S. Doctor of Pharmacy programs indicate that graduates are able to design and implement intervention strategies for individuals to improve health and wellness. Additionally, the Core Entrustable Professional Activities for New Pharmacy Graduates developed by the American Association of Colleges of Pharmacy give examples of tasks that pharmacy graduates must be able to perform without direct supervision upon entering practice indicating pharmacists are trained to "assess a patient's signs and symptoms to determine whether the patient can be treated within the scope of practice or requires a referral" and to "follow an evidence-based disease management protocol". ${ }^{27}$ 
Pharmacists and physicians have formally expressed few concerns with pharmacists ability to diagnose and treat acne. In surveys and in a multi-stakeholder process, neither pharmacists nor physicians rated acne as a condition of high concern with respect to pharmacist treatment in both the U.K. and Canada. ${ }^{28-}$ 30 In addition, Australian pharmacists were presented case vignettes to assess their readiness to treat certain conditions; pharmacists demonstrated they were able to select appropriate antibiotics for adolescent acne $100 \%$ of the time. ${ }^{31}$

Community pharmacists have successfully leveraged standardized protocols to manage and prescribe for minor conditions such as influenza, ${ }^{32}$ strep throat, ${ }^{33}$ and cold sores, ${ }^{34}$ among others. Such protocols assist with the identification of patients who are good candidates for treatment in the pharmacy versus patients who should be referred to other providers. Standardized protocols are already available for the treatment of acne by pharmacists. ${ }^{35}$ In general, pharmacist prescribing for acne is typically limited to mild acne. As a result, referral parameters tend to revolve around identifying more severe acne or other potential conditions (e.g., rosacea). In addition, referral is usually indicated if a patient has tried two topical products from the pharmacist without noted improvement in the condition, or if a patient is pregnant or breastfeeding.

Pharmacists have leveraged protocols in successfully treating patients with mild acne. Collectively, published studies from the UK and Canada show pharmacists have successfully treated more than 1,000 patients with acne over a 10 -year period. ${ }^{14-20}$ We found no published studies that reported any negative outcomes of acne patients treated by pharmacists. While the studies generally examined a broad range of pharmacisttreated minor ailments and did not break out data for acne separately, patients reported high rates of satisfaction with the care provided and reported high rates of symptomatic improvement. ${ }^{14,16}$

\section{Pharmacist Treatment Could Lead to Adverse Outcomes Such as Scarring}

As noted previously, published studies have found pharmacists have successfully managed and treated more than 1,000 patients with acne, with no reported negative health outcomes including scarring. It could be argued that pharmacist management could actually decrease adverse outcomes, if the accessibility of pharmacists reduces delays in treatment.

Indeed, patients delaying treatment of acne is reported as a frequent cause of scarring by dermatologists. ${ }^{36}$ Unfortunately, delaying acne treatment is common: studies have reported $40 \%$ to $51 \%$ of patients did not seek medical help, and another found $74 \%$ of patients waited over a year to seek medical assistance. ${ }^{37-38}$ In another study, the majority of respondents did not seek help from a physician because of either embarrassment or the belief that acne was not severe enough for medical assistance. ${ }^{13}$

Pharmacies may increase access to care for patients with mild acne given their convenience. In the United States, $90 \%$ of all Americans live within five miles of a pharmacy, and pharmacies also offer convenient hours of operation including nights, weekends, and holidays. ${ }^{39}$ In studies of pharmacist treatment of other minor ailments, patients report seeking care at pharmacies faster than other medical venues, and a high percentage of patients seek care on evenings or weekends. ${ }^{40}$

\section{Allowing pharmacists to prescribe could worsen antimicrobial resistance.}

Given that pharmacist management and treatment of acne is generally limited to mild acne, the development of antimicrobial resistance is unlikely from pharmacist prescribing. First, there has been a general shift towards non-antibiotic medications to treat mild acne. ${ }^{41}$ The clinical guidelines in both the U.S. and Europe recommend topical benzoyl peroxide or a retinoid as first-line therapy for acne. ${ }^{8,42}$ When antibiotics are used, topical products including clarithromycin and erythromycin are recommended for mild acne. Neither guideline supports use of these agents as monotherapy and the guidelines recommend combination with benzoyl peroxide as a strategy to minimize antibiotic resistant organisms. ${ }^{8,42-43}$

Pharmacists have a long history of successfully engaging in outpatient antimicrobial stewardship initiatives generally, and in the treatment of minor ailments specifically. ${ }^{44}$ Pharmacists have demonstrated higher adherence to guideline-based prescribing than other health professions, and thus, it could be argued pharmacists may actually decrease antimicrobial resistance related to mild acne. ${ }^{45}$

\section{This will fragment care delivery and it is inconsistent with the Patient-Centered Medical Home (PCMH) model of care.}

Care provided by urgent care facilities, emergency departments and other venues has the potential to lead to what some consider "fragmentation of care." Jurisdictions have remedied this by requiring notification of care provided by a pharmacist within a specified timeframe (5 days in Idaho, for example), although pharmacists are often alone in closing this communication gap. ${ }^{46}$ Further, care cannot be fragmented if it is never provided; as previously reported, a significant portion of acne patients either delay or never seek medical care. By enabling treatment at pharmacies, patients can get treatment sooner and, therefore, connected to the medical team sooner.

A related concern is about pharmacist access to the electronic medical record (EMR). In the U.S., this concern is not unique to pharmacy as it is rare for various healthcare points of access to utilize the same EMR (e.g. urgent care, private medical practices, etc.) and practitioners rely on patient history in order 
to provide care. While pharmacy EMR access is growing, pharmacists have demonstrated the ability to collect the relevant information necessary to diagnose and treat patients within their practice settings. To that end, a standardized data collection form is available for pharmacists. ${ }^{66}$ The form ascertains key parameters that may facilitate a referral. For example, by inquiring about the use of specific medications (e.g., glucocorticoids), it helps the pharmacist identify if the acne may be the result of, or worsened by, certain medications. ${ }^{67}$

\section{Conclusion}

Pharmacist management of acne with use of a standardized protocol has been tested and has proven successful over at least a decade in the U.S., U.K., and Canada. Studies have found the model to be safe and effective, while simultaneously increasing more timely access to care for patients. Other jurisdictions considering this service should allow the available evidence to inform the inevitable policy debates.

Funding Support: None

Conflicts of Interest: None

Disclaimer: The views expressed in this manuscript are those of the authors alone, and do not necessarily reflect those of their respective employers.

\section{References}

1. Titus S, Hodge J. Diagnosis and Treatment of Acne. Am Fam Physician. 2012;86(8):734-740. Available from: https://www.aafp.org/afp/2012/1015/p734.pdf (Accessed November 8, 2020)

2. American Academy of Dermatology. Skin conditions by the numbers. Available from: https://www.aad.org/media/stats/conditions/skinconditions-by-the-numbers (Accessed November 8, 2020)

3. James WD. Clinical practice. Acne. N Engl J Med 2005; 352:146372.

4. American Academy of Dermatology. Acne: Signs and Symptoms. Available from: https://www.aad.org/public/diseases/acne-androsacea/acne\#symptoms (Accessed November 8, 2020)

5. Tan JK, Tang J, Fung K, et al. Development and validation of a comprehensive acne severity scale. J Cutan Med Surg 2007;11:2116.

6. Layton AM, Henderson CA, Cunliffe WJ. A clinical evaluation of acne scarring and its incidence. Clin Exp Dermatol 1994;19:3038.

7. Jacob Cl, Dover JS, Kaminer MS. Acne scarring: A classification system and review of treatment options. J Am Acad Dermatol. 2001;45(1):109-117. https://doi.org/10.1067/mjd.2001.113451

8. Zaenglein AL, Pathy AL, Schlosser BJ, et al. Guidelines of care for the management of acne vulgaris. J Am Acad Dermatol. 2016;74(5):945-973. Doi: 10.1016/j.jaad.2015.12.037

9. American Academy of Dermatology/Milliman. Burden of Skin Disease. 2017. Available from: www.aad.org/BSD. (Accessed November 22, 2018)
10. Coret KC, Cheng CE, Irwin B, Kimball AB. Self-Reported Help-Seeking Behaviors and Treatment Choices of Adolescents Regarding Acne. Pediatric Dermatology. 2013;30(1):36-41.

11. Tan JKL, Vasey K, Fung KY. Beliefs and perceptions of patients with acne. J Am Acad Dermatol. 2001;44(3):439445.

12. Chernyshov PV, Petrenko A, Kopylova V. What Healthrelated Quality of Life Factors Influence the Decision of Patients with Acne to Visit a Dermatologist? J Clin Aesthet Dermatol. 2018;11(7):21-25.

13. Desai KP, Martyn-Simmons C, Viner R, Segal T. Help-seeking behaviors, opportunistic treatment and psychological implications of adolescent acne: cross-sectional studies in schools and hospitals outpatient departments in the UK. BMJ Open. 2017;7:e016964. Doi: 10.1136/bmjopen-2017016964

14. Taylor JG, Mansell K. Patient Feedback on Pharmacist Prescribing for Minor Ailments in a Canadian Province. Inov Pharm. 2017;8(1): Article 17. http://pubs.lib.umn.edu/innovations/vol8/iss1/17 DOI: https://doi.org/10.24926/21550417.1331 (Accessed November 8, 2020)

15. Taylor JG, Joubert R. Pharmacist-led minor ailment programs: a Canadian perspective. Int J Gen Med. 2016;9:291-302. DOI: 10.2147/IJGM.S99540

16. Mansell K, Bootsman N, Kuntz A, Talyor J. Evaluating pharmacist prescribing for minor ailments. International Journal of Pharmacy Practice. 205;23:95-101. DOI: 10.1111/ijpp.12128

17. Fielding S, Porteous T, Ferguson J, et al. Estimating the burden of minor ailment consultations in general practices and emergency departments through retrospective review of routine data in North East Scotland. Family Practice. 2015;32(2):165-172.

18. Mondkar A, Wright D, Patel R, et al. NHS England London Region - Minor Ailments Scheme (MAS) review. December 2017.

19. Gray N. Evaluation of the Bolton 'Pharmacy First' Minor Ailments Service. December 2008.

20. Jones I. Evaluation of the Choose Pharmacy common ailments service: Final report. Welsh Government Social Research. July 2015. Available from:

http://www.primarycareone.wales.nhs.uk/sitesplus/docum ents/1191/Evaluation\%20of\%20the\%20Choose\%20Pharma cy\%20Common\%20Ailments\%20Service\%20\%28Final\%20R eport\%202015\%29.pdf (Accessed November 8, 2020)

21. Adams AJ, Chopski NL. Rethinking Pharmacy Regulation: Core Elements of Idaho's Transition to a "Standard of Care" Approach. J Am Pharm Assoc. 2020;60(6):E109-E112. https://doi.org/10.1016/i.japh.2020.07.013

22. Adams AJ. Pharmacist Prescriptive Authority: Lessons from Idaho. Pharmacy 2020;8(3):112. Heeps://doi.org/10.3390/pharmacy8030112

23. Florida Board of Pharmacy. 64B8-36.003. Medicinal Drugs Which May Be Ordered by Pharmacists. Available from: https://www.flrules.org/gateway/RuleNo.asp?title=PRESCR IPTIONS\%200F\%20CERTAIN\%20MEDICINAL\%20DRUGS\%20 BY\%20PHARMACISTS\&ID=64B8-36.003 (Accessed November 8, 2020) 
24. Krakowski AC, Eichenfield LF. Pediatric acne: clinical presentations, evalutation, and management. J Drugs Dermatol. 2007;6(6):589-93.

25. Gold MH, Andriessen A, Biron J. Self-diagnosis of Mild-toModerate Acne for Self Treatment with Blue Light Therapy. J Clin Aesthet Dermatol. 2009;2(4):40-44.

26. Menon C, Gipson K, Bowe WP, et al. Validity of subject selfreport for acne. Dermatology. 2008;317(2):164-168. Doi: 10.1159/000136655

27. Haines ST, Pittenger AL, Stolte SK, et al. Core entrustable professional activities for new pharmacy graduates. Am J Pharm Educ. 2017;81(1):Article S2

28. Taylor J, Mansell K. Minor ailment prescribing: part I pharmacist feedback. Self Care. 2016;7:10-21.

29. Taylor J. Minor ailment prescribing: part II - physician feedback. Self Care. 2016;7:22-40.

30. Nazar H, Nazar Z, Yeung A. Consensus methodology to determine minor ailments appropriate to be directed for management within community pharmacy. Res Social Adm Pharm. 2018;14(11):1027-1042. Doi: 10.1016/j.sapharm.2018.01.001

31. Ung E, Czarniak $P$, Sunderland B, et al. Assessing pharmacists' readiness to prescribe oral antibiotics for limited infections using a case-vignette technique. Int J Clin Pharm. 2017;31:61-69.

32. Klepser ME, Adams AJ. Pharmacy-based management of influenza: lessons learned from research. International Jounral of Pharmacy Practice. 2018. https://doi.org/10.1111/iipp.12488

33. Klepser, ME, Adams, AJ, Klepser, DG. Antimicrobial stewardship in outpatient settings: Leveraging innovative physician-pharmacist collaborations to reduce antibiotic resistance. Health Security, 13, 166-73. DOI: 10.1089/hs.2014.0083

34. Adams AJ, Klepser ME. Pharmacy-Based Assessment and Management of Herpes Labialis (Cold Sores) with Antiviral Therapy. Innovations in Pharmacy. 11(3):2020. https://doi.org/10.24926/iip.v11i3.1532 (Accessed November 8, 2020)

35. MedSask. Guidelines for Prescribing for Minor Ailments and Patient Self-Care. Available from: https://medsask.usask.ca/professional-practice/minorailment-guidelines.php (Accessed November 8, 2020)

36. Madden WS, Landells ID, Poulin Y, et al. Treatment of acne vulgaris and prevention of acne scarring: Canadian consensus guidelines. J Cutaneous Medicine and Surgery. 2000;4(1):S4-13. https://doi.org/10.1177/12034754000040S102
37. Brazier Y. Acne: what causes it and how to relieve it. MedicalNewsToday. November 5, 2015. Available from: https://www.medicalnewstoday.com/articles/302002.php (Accessed November 8, 2020)

38. Pawin $\mathrm{H}$, Chivot M, Beylot $\mathrm{C}$, et al. Living with Acne. A Study of Adolescents' Personal Experiences. Dermatology. 2007;215:308-314. DOI: 10.1159/000107624

39. Roberts GE, Rubin SE, Smith JK, et al. Public Health Perceptions of Community Pharmacy Partnership Opportunities. Journal of Public Health Management and Practice. 2015;21(4):413-415. Doi: 10.1097/PHH.0000000000000276

40. Taitel M, Cohen E, Terranova B, et al. Pharmacists as Immunization Providers: Patient Attitudes and Perceptions. Pharmacy Times. Available from:

http://www.pharmacytimes.com/publications/issue/2011/ September2011/Pharmacists-as-Immunization-ProvidersPatient-Attitudes-and-Perceptions/ (November 8, 2020)

41. Thevarajah S, Balkrishnan R, Camacho FT, et al. Trends in prescription of acne medication in the US: shift from antibiotic to non-antibiotic treatment. J Dermatolog Treat. 2005;16(4):224-228.

42. Nast A, Dréno B, Bettoli $V$, et al. European evidence-based (S3) guideline for the treatment of acne - update 2016 short version. J Eur Acad Dermatol Venereol. 2016;30(8):1261-1268.

43. Williams HC, Dellavelle RP, Garner S. Acne vulgaris. The Lancet. 2012;379(9813):361-372.

44. Dobson EL, Klepser ME, Pogue JM, et al. Outpatient antibiotic stewardship: Interventions and opportunities. J Am Pharm Assoc. 2017;57(4):464-473.

45. Beahm NP, Smyth DJ, Tsuyuki RT. Outcomes of Urinary Tract Infection Management by Pharmacists (RxOUTMAP). Can Pharm J. 2018;151(5):305-314.

46. Tsuyuki RT. FAQs (frequent asinine questions) on pharmacists' scope of practice. Can Pharm J. 2018;151(4):212-213.

47. MedSask. Pharmacist Assessment - Mild Acne. Available from:

https://medsask.usask.ca/documents/pdfs/Acne assessme nt.pdf (Accessed November 8, 2020)

48. Truter I. Evidence-Based Pharmacy Practice: Acne Vulgaris. South African Pharmaceutical Journal. April 2009;12-19. 